PROCEEDINGS OF THE

AMERICAN MATHEMATICAL SOCIETY

Volume 137, Number 11, November 2009, Pages 3563-3567

S 0002-9939(09)09982-1

Article electronically published on June 18, 2009

\title{
SIGNS OF FOURIER COEFFICIENTS OF TWO CUSP FORMS OF DIFFERENT WEIGHTS
}

\author{
WINFRIED KOHNEN AND JYOTI SENGUPTA
}

(Communicated by Wen-Ching Winnie Li)

\begin{abstract}
We investigate sign changes of Fourier coefficients of cusp forms
\end{abstract} of different weights.

\section{INTRODUCTION}

Signs of Fourier coefficients or Hecke eigenvalues of cusp forms in one and several variables have been recently studied in various aspects; for a survey see for example 1 .

In this note we will investigate the signs of the Fourier coefficients $a(n)$ and $b(n)(n \in \mathbf{N})$ of two non-zero cusp forms $f$ and $g$ of different weights $k_{1}$ and $k_{2}$ at least 2 , respectively, and level $N$. If $a(n)$ and $b(n)$ are totally real algebraic numbers for all $n$ and $a(1)=b(1)=1$, then we will show that up to the action of a Galois automorphism, infinitely many of the $a(n)$ have the same sign (respectively opposite sign) as the corresponding $b(n)$. The main ingredients in the proof are the analytic properties of the Rankin-Selberg zeta function attached to $f$ and $g$, a classical theorem of Landau on Dirichlet series with non-negative coefficients and the "bounded denominators" argument in the theory of modular forms.

As an amusing and rather immediate corollary one obtains that the generating function of the numbers $h(n) a(n)(n \geq 1)$ never is a cusp form of any even weight $\geq 2$ and any level. Here $h$ is any function on the positive integers that takes rational values, is of polynomial growth and such that $h(1)=1, h(n)>0$ for $n \gg 1$ and $h(n) \gg n^{c}$ for some $c>0$ whenever $n$ is large. (We in fact shall prove a slightly more general statement.) Of course, this statement is believed to be morally true by anyone, but a priori it seems not so clear how to produce a formally correct and simple proof of it.

Notation. For $z \in \mathcal{H}$ the complex upper half-plane, we set $q:=e^{2 \pi i z}$.

For an integer $k$ and $N$ a natural number we denote by $S_{k}(N)$ the space of cusp forms of weight $k$ on the group $\Gamma_{0}(N)$ consisting of matrices in $S L_{2}(\mathbf{Z})$ with lower left component divisible by $N$. We will always suppose that $k \geq 2$.

Received by the editors September 23, 2008.

2000 Mathematics Subject Classification. Primary 11F30.

(C)2009 American Mathematical Society Reverts to public domain 28 years from publication 
For $f, g \in S_{k}(N)$ we let

$$
\langle f, g\rangle=\int_{\Gamma_{0}(N) \backslash \mathcal{H}} f(z) \overline{g(z)} y^{k-2} d x d y \quad(z=x+i y)
$$

be the Petersson scalar product of $f$ and $g$.

If $f(z)=\sum_{n>1} a(n) q^{n} \in S_{k}(N)$ and $\rho \in \operatorname{Gal}(\overline{\mathbf{Q}} / \mathbf{Q})$, we set $f^{\rho}(z):=$ $\sum_{n \geq 1} a(n)^{\rho} q^{n}$. Then $f^{\rho} \in S_{k}(N)$, as is well known. Indeed, this follows from the fact that $S_{k}(N)$ has a basis consisting of functions with rational Fourier coefficients [3, Thm. 3.5.2].

If $s$ is a complex number, we denote by $\sigma$ its real part.

\section{Statement of Results}

Theorem. Let $f(z)=\sum_{n>1} a(n) q^{n} \in S_{k_{1}}(N)$ and $g(z)=\sum_{n>1} b(n) q^{n} \in S_{k_{2}}(N)$ with $k_{1} \neq k_{2}$ and suppose that $a(n)$ and $b(n)$ are totally real algebraic numbers for all $n \geq 1$ and $f$ and $g$ are normalized, i.e. $a(1)=b(1)=1$. Let $\epsilon \in\{ \pm 1\}$. Then there exists $\rho \in \operatorname{Gal}(\overline{\mathbf{Q}} / \mathbf{Q})$ and a sequence of natural numbers $\left(n_{\nu}\right)_{\nu \in \mathbf{N}}$ such that $a\left(n_{\nu}\right) \neq 0, b\left(n_{\nu}\right) \neq 0$ and sign $a\left(n_{\nu}\right)^{\rho}=\epsilon$ sign $b\left(n_{\nu}\right)^{\rho}$ for all $\nu$.

Remark. Note that the assumptions of the Theorem are satisfied if $f$ and $g$ are normalized Hecke eigenforms in the corresponding subspaces of newforms of level $N$.

Corollary. Let $f(z)=\sum_{n \geq 1} a(n) q^{n} \in S_{k}(N)$ with a $(n)$ totally real for all $n \geq 1$ and $a(1)=1$. Let $h: \mathbf{N} \rightarrow \mathbf{R}$ be a function that is of polynomial growth and takes totally real, totally positive algebraic numbers for $n \gg 1$. Assume further that $h(1)=1$ and that there exists $\rho_{0} \in \operatorname{Gal}(\overline{\mathbf{Q}} / \mathbf{Q})$ such that $h(n)^{\rho_{0}} \gg n^{c}$ for some $c>0$ whenever $n$ is large. Then the series

$$
\sum_{n \geq 1} h(n) a(n) q^{n}
$$

is not a cusp form in $S_{\ell}(M)$, for any $\ell \geq 2$ and any $M \in \mathbf{N}$.

\section{Proofs}

Proof of Theorem. The space $S_{k}(N)$ has a basis of functions each of which is obtained (by applying the standard $U$ - and $V$-operators in Atkin-Lehner theory) from a unique normalized Hecke eigenform that is a newform of exact level a divisor of $N$. Furthermore, the field obtained from $\mathbf{Q}$ by adjoining the Fourier coefficients of such a Hecke eigenform is a number field, i.e. is of finite degree over $\mathbf{Q}$, and is totally real. If for any $\rho \in \operatorname{Gal}(\overline{\mathbf{Q}} / \mathbf{Q})$ we write $f^{\rho}$ and $g^{\rho}$ in terms of these corresponding bases, the coefficients in these bases are real algebraic numbers due to our assumption on $a(n)$ and $b(n)$; hence

$$
K_{f, g}:=\mathbf{Q}\left(\{a(n), b(n)\}_{n \geq 1}\right)
$$

is also a totally real number field.

We let $G$ be the set of embeddings of $K_{f, g}$ over $\mathbf{Q}$ into $\mathbf{R}$.

We will only treat the case $\epsilon=-1$; the other case works in a similar way, mutatis mutandis. Since $G$ is finite it is sufficient to show that there exists a sequence $\left(n_{\nu}\right)_{\nu \in \mathbf{N}}$ in $\mathbf{N}$ and for each $n_{\nu}$ there exists an element $\rho_{\nu} \in G$ such that $\left(a\left(n_{\nu}\right) b\left(n_{\nu}\right)\right)^{\rho_{\nu}}<0$. 
Assume that this would not be true. Then

$$
(a(n) b(n))^{\rho} \geq 0
$$

for all $n$ large enough, say for $n \geq n_{0}$ and all $\rho \in G$.

Let $p_{1}, \ldots, p_{r}$ be the different prime numbers less than $n_{0}$ and put

$$
M:=p_{1} \ldots p_{r} .
$$

Since $\left((a(1) b(1))^{\rho}=1>0\right.$ for all $\rho \in G$, we conclude that

$$
(a(n) b(n))^{\rho} \geq 0
$$

for all $n$ with $\operatorname{gcd}(n, M)=1$ and all $\rho \in G$. In particular, if

$$
c(n):=\operatorname{tr}_{K_{f, g} / \mathbf{Q}}(a(n) b(n)) \quad(n \in \mathbf{N}),
$$

then $c(n) \geq 0$ for all $n$ with $\operatorname{gcd}(n, M)=1$.

Let us denote by $f_{M}^{\rho}$ and $g_{M}^{\rho}$ the series obtained from $f^{\rho}$ and $g^{\rho}$, respectively, by restricting the summation to those $n$ with $\operatorname{gcd}(n, M)=1$. Then, as is well-known, $f_{M}^{\rho} \in S_{k_{1}}\left(N M^{2}\right)$ and $g_{M}^{\rho} \in S_{k_{2}}\left(N M^{2}\right)$.

Let

$$
R_{f_{M}^{\rho}, g_{M}^{\rho}}(s):=\sum_{n \geq 1, g c d(n, M)=1} a(n)^{\rho} b(n)^{\rho} n^{-s} \quad(\sigma \gg 1)
$$

be the Rankin-Selberg Dirichlet series attached to $f_{M}^{\rho}$ and $g_{M}^{\rho}$ and suppose without loss of generality that $k_{1}>k_{2}$. If we set

$R_{f_{M}^{\rho}, g_{M}^{\rho}}^{*}(s):=(2 \pi)^{-2 s} \Gamma(s) \Gamma\left(s-k_{2}+1\right) \zeta_{N M^{2}}\left(2 s-\left(k_{1}+k_{2}\right)+2\right) R_{f_{M}^{\rho}, g_{M}^{\rho}}(s) \quad(\sigma \gg 1)$, where

$$
\zeta_{N M^{2}}(s):=\prod_{p \mid N M^{2}}\left(1-p^{-s}\right) \cdot \zeta(s),
$$

then, as is well-known, $R_{f_{M}^{\rho}}^{*}, g_{M}^{\rho}(s)$ extends to an entire function on C. Indeed, by the Rankin-Selberg method one has the integral representation

$R_{f_{M}^{\rho}, g_{M}^{\rho}}(s)=\int_{\Gamma_{0}(A) \backslash \mathcal{H}} f_{M}^{\rho}(z) \overline{g_{M}^{\rho}(z)} E_{k_{1}-k_{2}}^{*}\left(z ; s-k_{1}+1\right) y^{k_{1}-2} d x d y \quad(z=x+i y)$.

Here $A:=N M^{2}$ and for $k$ a non-negative integer we have put

$$
E_{k}^{*}(z ; s):=\pi^{-s} \Gamma(s+k) E_{k}(z ; s),
$$

where

$$
E_{k}(z ; s):=\zeta(2 s+k) \sum_{\gamma=\left(\begin{array}{c}
\dot{c} \dot{d} \\
\dot{2}
\end{array}\right) \in \Gamma_{0}(A)_{\infty} \backslash \Gamma_{0}(A)} \frac{y^{s}}{(c z+d)^{k}|c z+d|^{2 s}} \quad(z \in \mathcal{H} ; s \in \mathbf{C}, \sigma \gg 1)
$$

is the non-holomorphic Eisenstein series of weight $k>0$ and level $A$ for the cusp $i \infty$ and

$$
\Gamma_{0}(A)_{\infty}=\left\{ \pm 1\left(\begin{array}{cc}
1 & m \\
0 & 1
\end{array}\right) \mid m \in \mathbf{Z}\right\}
$$

Note that for $k>0$ the function $E_{k}^{*}(z ; s)$ extends to an entire function in $s$; cf. e.g. 2], Cor. 7.2.11, p. 286, with $\chi$ resp. $\psi$ the trivial character modulo 1 resp. modulo $A$ and $z$ replaced by $A z$ in the notation there.

Note that for $k=0$ the Eisenstein series has a pole at $s=1$ (loc. cit.), and so in the equal weight case $k_{1}=k_{2}$ the function $R_{f_{M}^{\rho}, g_{M}^{\rho}}(s)$ has a possible pole at $s=k_{1}$ of residue essentially equal to $\left\langle f_{M}^{\rho}, g_{M}^{\rho}\right\rangle$. Hence our results probably would 
have some extension also to the case $k_{1}=k_{2}$ if in addition in this case one assumes some orthogonality conditions for $f$ and $g$ along with all their conjugates.

We put

$$
R(s):=\sum_{n \geq 1, g c d(n, M)=1} c(n) n^{-s} \quad(\sigma \gg 1)
$$

and

$$
R^{*}(s):=(2 \pi)^{-2 s} \Gamma(s) \Gamma\left(s-k_{2}+1\right) \zeta_{N M^{2}}\left(2 s-\left(k_{1}+k_{2}\right)+2\right) R(s) \quad(\sigma \gg 1) .
$$

Then $R^{*}(s)$ extends holomorphically to $\mathbf{C}$.

Denote the coefficients of the Dirichlet series

$$
\zeta_{N M^{2}}\left(2 s-\left(k_{1}+k_{2}\right)+2\right) R(s) \quad(\sigma \gg 1)
$$

by $e(n)(n \in \mathbf{N})$. Since the coefficients of $R(s)$ are non-negative, we have $e(n) \geq 0$ for all $n \in \mathbf{N}$. (Note that if we only had $c(n) \geq 0$ for all but a finite number of $n$, then in general we could not conclude that $e(n) \geq 0$ for all but a finite number of n.)

By a well-known and classical theorem of Landau we now find that

$$
\sum_{n \geq 1} e(n) n^{-s} \quad(\sigma \gg 1)
$$

must either have a singularity at the real point of its abscissa of convergence or must converge for all $s \in \mathbf{C}$. Since (1) has holomorphic continuation to $\mathbf{C}$, the first alternative is excluded.

In particular we conclude that

$$
e(n) \ll_{A} n^{A}
$$

for all negative $A$.

We now invoke the "bounded denominators" argument [3, Thm. 3.5.2]. Since $a(n)$ and $b(n)$ are algebraic for all $n$, there exist non-zero integers $D_{1}$ and $D_{2}$ such that $D_{1} a(n)$ and $D_{2} b(n)$ are in $\mathcal{O}$, the ring of algebraic integers of $\overline{\mathbf{Q}}$, for all $n \geq 1$. It follows that $\left(D_{1} a(n)\right)^{\rho},\left(D_{2} b(n)\right)^{\rho} \in \mathcal{O}$; hence $\left(D_{1} D_{2} a(n) b(n)\right)^{\rho} \in \mathcal{O}$ for all $\rho \in G$ and $n \in \mathbf{N}$. From this we conclude that $D_{1} D_{2} c(n) \in \mathbf{Z}$ for all $n$; hence also $D_{1} D_{2} e(n) \in \mathbf{Z}$ for all $n$.

From (2) (say with $A=-1$ ) we now find that $e(n)=0$ for $n \gg 1$. But

$$
(2 \pi)^{-2 s} \Gamma(s) \Gamma\left(s-k_{2}+2\right) \sum_{n \geq 1} e(n) n^{-s} \quad(\sigma \gg 1)
$$

extends to an entire function as we noted above. Since $\Gamma(s) \Gamma\left(s-k_{2}+2\right)$ has poles at $s=0,-1,-2, \ldots$ we see that the Dirichlet polynomial $\sum_{n \geq 1} e(n) n^{-s}(s \in \mathbf{C})$ must vanish at these points, and we obtain a linear system of equations for the $e(n)(n \ll 1)$ whose determinant is a Vandermonde determinant, hence non-zero. It follows that $e(n)=0$; hence $c(n)=0$ for all $n \geq 1$, which contradicts $c(1)=|G|$.

This concludes the proof of the Theorem.

Proof of Corollary. We put

$$
g(z):=\sum_{n \geq 1} h(n) a(n) q^{n} \quad(z \in \mathcal{H})
$$


and suppose that $g \in S_{\ell}(M)$ for some $\ell \geq 2$ and $M \in \mathbf{N}$. First assume that $\ell \neq k$. Then by the Theorem with $\epsilon=-1$ and $N$ replaced by $\operatorname{lcm}(N, M)$, there exist $\rho \in \operatorname{Gal}(\overline{\mathbf{Q}} / \mathbf{Q})$ and $n \in \mathbf{N}, n$ arbitrarily large, such that $a(n) \neq 0$ and

$$
\operatorname{sign}(h(n) a(n))^{\rho}=-\operatorname{sign} a(n)^{\rho} .
$$

Since for $n$ large, $h(n)^{\rho}>0$, we obtain a contradiction.

Now suppose that $\ell=k$. Since $g \in S_{k}(M)$, also $g^{\rho_{0}} \in S_{k}(M)$, and by Deligne's bound we have

$$
(h(n) a(n))^{\rho_{0}} \ll_{\epsilon} n^{(k-1) / 2+\epsilon} \quad(\epsilon>0) .
$$

Taking $\epsilon=c$ and observing our assumption $h(n)^{\rho_{0}} \gg n^{c}$, it follows that

$$
a(n)^{\rho_{0}} \ll n^{(k-1) / 2} .
$$

On the other hand, by a classical and well-known result of Rankin one has

$$
\lim \sup _{n \rightarrow \infty} \frac{|a(n)|_{0}}{n^{(k-1) / 2}}=\infty,
$$

a contradiction. This proves our claim.

\section{REFERENCES}

[1] W. Kohnen, Sign changes of Fourier coefficients and eigenvalues of cusp forms. In: Number Theory, Proc. 4th China-Japan Seminar (eds.: S. Kanemitsu and J.-Y. Liu), pp. 97-107. World Scientific Publ. Company, 2007. MR2364838(2008m:11099)

[2] T. Miyake, Modular Forms, Springer, Berlin-Heidelberg-New York, 1989. MR.1021004 (90m:11062)

[3] G. Shimura, Introduction to the arithmetic theory of automorphic functions, Iwanami Shoten and Princeton Univ. Press, 1971. MR 0314766 (47:3318)

Mathematisches Institut, INF 228, Universität Heidelberg, D-69120 Heidelberg, GERMANY

E-mail address: winfried@mathi.uni-heidelberg.de

School of Mathematics, Tata Institute of Fundamental Research, Homi Bhabha RoAD, 400005 Mumbai, India

E-mail address: sengupta@math.tifr.res.in 